\title{
Proyecto Escuela: Espacio de Paz. Reflexiones sobre una Experiencia en un Centro Educativo
}

\section{School Project: “Area of Peace”. Thoughts about an Experience in an Educative Centre}

\author{
José Manuel de Oña Cots ${ }^{1 *}$ \\ Emilio Andrés García Gálvez ${ }^{2}$ \\ ${ }^{1}$ Universidad de Málaga ${ }^{2}$ Ayuntamiento de Málaga
}

\begin{abstract}
Se enmarca esta investigación dentro del Master Interuniversitario de Cultura de Paz, Conflictos, Educación y Derechos Humanos. La finalidad principal de la misma es conocer la situación del Proyecto "Red Escuela: Espacio de paz" en un IES de la provincia de Málaga, por medio de las valoraciones y perspectivas del alumnado que acude al Aula de Convivencia y al análisis de las hojas de incidencia que supervisan los docentes a cargo de este aula. Exponemos una investigación de carácter exploratorio y descriptivo en la que se utilizó tanto la observación no participante, como el análisis de contenidos y un cuestionario con preguntas abiertas, cerradas y de tipo Likert. Los participantes son 206 alumnos repartidos en $1^{\circ}$ de ESO (117) y $2^{\circ}$ de ESO (89). Los resultados que se muestran corresponden al análisis de algunos ítems y respuestas seleccionadas del cuestionario referidos al Aula de Convivencia y a la hoja de incidencias, categorizadas las causas por las que eran enviados allí en "Interrupciones", "Violencia", "Conflicto" o "Falta de respeto", entre otras. Los datos expuestos nos ofrecen una doble realidad: por un lado los sujetos del estudio parecen tener bien interiorizado el sentido teórico del proyecto, pero a la hora de concretarlo en la práctica diaria, la situación se aleja de lo que debería esperarse de un proyecto de estas características, convirtiéndose en un espacio punitivo y de reducción de conductas.
\end{abstract}

Descriptores: Educación, Convivencia, Escuela, Paz, Cultura de paz, Educación para la paz.

This research started as part of the final project for the Culture of Peace, Conflict, Education and Human Rights Interuniversity Master degree in Malaga. The main purpose of this paper is to describe and to interpret the situation of the Project "Red Escuela: Espacio de Paz" in a high school from Málaga city, through high school students' conceptions and the analysis of the papers filled out by the students who have been sent to the Coexistance classroom. This is an exploratory and descriptive research in which we use both non-participant observation and content analysis and a questionnaire with open and closed questions, as Likert questions. The 206 participants are divided into 117 students of 1 st year and 89 of 2nd year. The results shown belong to the analysis of the questionnaire items related to classroom Coexistence, focused on categories such as "Interruptions", "Violence", "Conflict" or "Disrespect". The presented data show a double reality: first students appear to have so internalized the theoretical sense of the project, but at the time, when these attitudes are expected to be included in daily practice, the situation is far from what should be got of a project of these characteristics.

Keywords: Education, Coexistence, School, Peace, Culture of peace, Education for peace.

*Contacto: josecots@uma.es

ISSN: $1696-4713$

www.rinace.net/reice/
Recibido: $\quad 14$ de abril 2015

$1^{\text {a }}$ Evaluación: 30 de julio 2015

$2^{\text {a }}$ Evaluación: 15 de septiembre 2015

Aceptado: $\quad 14$ de octubre 2015 


\section{Introducción}

Vivimos en un mundo que se nos presenta como un auténtico desafío para la educación, entendida como herramienta para transformar la realidad. Un mundo cargado de conflictos, profundos cambios sociales, y plagado de situaciones que ponen en cuestión la dignidad de la persona y nuestra capacidad de convivir.

En este contexto, la educación para la paz viene a presentarse como una posibilidad educativa para la construcción común de otra cultura, una en la que todos podamos aportar y se rechace la violencia como forma de solución de los problemas; que opte por estilos dialógicos y de negociación entre personas y pueblos. La necesidad de plantear una cultura de paz que nos comprometa política y culturalmente con la dignidad de la persona, y que nos ayude a rescatar valores como la tolerancia y la solidaridad, es hoy un reto urgente para todos, ya que está en juego el respeto a los derechos fundamentales de la persona.

Tal y como hemos señalado anteriormente, nuestra investigación se enmarca en el contexto del Máster Interuniversitario de Cultura de Paz, Conflictos, Educación y Derechos Humanos, y pretende exponer la situación del Proyecto "Red Escuela: Espacio de Paz" en un Centro de Secundaria de la provincia de Málaga, visto básicamente desde la perspectiva del alumnado, ya que tratamos de dar la voz a los auténticos protagonistas del trabajo educativo, por medio de sus valoraciones y opiniones. Además, hemos considerado la hoja de incidencia, documento que debía ser rellenado por cada alumno y alumna enviado al Aula de Convivencia, como parte esencial de nuestro trabajo en el sentido de dotarnos de claves importantes sobre el terreno respecto a qué se hace y cómo se actúa en el centro en relación al proyecto.

\section{La Escuela como Espacio de Paz}

El término "paz" es utilizado con mucha frecuencia hoy día, aunque se hace a veces de manera ambigua, como si se tratara de algo que únicamente viven personas o grupos determinados: pacifistas, voluntarios, etc. Muñoz y López (1999) nos han señalado cómo este concepto ha ido evolucionando a lo largo de la historia: desde una idea de paz como ausencia de guerra se va avanzando hacia otra muy relacionada con la construcción de condiciones sociales de justicia y respeto a los derechos de las personas, buscando estrategias para solucionar los conflictos que se produzcan e investigando sobre las dificultades que las personas tenemos para llegar a acuerdos pacíficos de convivencia (Sánchez, 2012). En esta evolución surge la idea de paz imperfecta (Muñoz, 2001), concepto que puede ayudarnos a entender la existencia del gran número de situaciones y propuestas pacíficas que se dan para solucionar los conflictos desde la no violencia, así como continuar promoviendo esa paz aun en lugares donde la violencia es excesivamente cotidiana.

Tomando como referencia lo expuesto, entendemos el concepto de "paz" como el ejercicio diario, siempre inacabado, en el que asumimos la igualdad de todos los seres en dignidad, el reconocimiento mutuo de los derechos, y la voluntad de construir juntos una ciudadanía común, encarando los conflictos de manera dialógica (Mayor, 2011). En palabras de Max-Neff (1998): "La paz es el desarrollo de capacidades humanas e interacciones causales" (p. 40). 
Esta necesaria construcción de una paz cada vez más implementada ha de llevarnos a que nos situemos ante la realidad de una forma determinada, una visión que dote de sentido y posibilidad a dicha construcción: el punto de partida de esta paz se encuentra en la capacidad del ser humano para crecer, optimizar y optimizarse, ir siendo persona en sociedad, junto a otros (Freire, 1996). Las personas están abocadas a la acción, al hecho de hacerse, ya que somos seres siempre incompletos, inacabados, siempre perfeccionables y siempre exigidos en ese hecho constructivo de ser persona y hacer sociedad (Ruiz, 2008). Y en esta construcción permanente (Flecha, 2006), la paz ha de convertirse en un valor para la ciudadanía, un valor que nos ayude a la promoción de una verdadera cultura de paz, en la que cada persona tenga la posibilidad de crecer personal y comunitariamente (Tuvilla, 2004).

Hablamos, en definitiva, de la promoción de una cultura sustentada en una serie de valores, actitudes y comportamientos que rechacen la violencia y prevengan y afronten los conflictos, tratando de atajar sus causas para solucionar los problemas mediante el diálogo y la negociación entre las personas, los grupos y las naciones (Naciones Unidas, 1998). Esto supone la necesidad de buscar un cambio de mentalidad que nos ayude a organizarnos en torno a la idea de la defensa del bien común, apoyada en algunos valores, entre otros, los que abordamos a continuación:

La dignidad de la persona como eje vertebrador de toda acción. Todos los seres humanos nacemos libres e iguales en dignidad y derechos, teniendo la posibilidad de utilizar la razón y la conciencia para vivir de manera fraternal unos con otros (Declaración Universal de los Derechos Humanos, Art. 1). Tal y como venimos sosteniendo, el ser humano es un ser social, lo relacional es parte intrínseca de lo humano, y encuentra su razón de ser en la construcción común de una sociedad cimentada en lo comunitario, en lo fraterno. La relación entre el individuo y la sociedad es de mutua necesidad para el desarrollo armónico de ambos.

Además de ser social, el ser humano es también un ser integral, posee múltiples facetas interrelacionadas entre sí. Una integralidad que se traduce en una realidad singular e irrepetible y que se va haciendo realidad en el encuentro, en la relación con sus iguales; ya que tanto la persona como la sociedad están siempre haciéndose, no acaban de estar nunca completas.

Convertir la defensa de esta dignidad y lo que significa en piedra angular de la cultura de paz es una responsabilidad que es necesario asumir para la cimentación de una sociedad sana, que reconozca las individualidades de cada sujeto y posibilite la participación de todos en un proyecto común: "Se trata de mantener la prioridad de los individuos y sus derechos, pero no de un individuo en abstracto, sino de los individuos concretos, reales, existentes en su diversidad y complejidad“ (Fernández, 2003, p. 49).

En esta línea añadiremos un valor que entendemos fundamental en la construcción de la cultura de paz: La solidaridad, entendida como la convergencia de tres momentos que se complementan y alimentan entre sí: en primer lugar, es una reacción ante la injusticia y el sufrimiento de los demás; en segundo lugar es una determinación por embarcarse en los procesos que tratan de erradicar las causas de estas situaciones; y por último es un estilo de vida que pone en juego nuestras posibilidades (Aranguren, 2002). Buscar la vinculación entre las personas, promover el sentimiento de interdependencia, encontrar el sentido a la propia existencia por medio del encuentro y la relación con los demás, podrán ser facilitadores de esta vivencia de la solidaridad que estamos defendiendo, de 
este aprendizaje de ser persona en plenitud (Gimeno, 2001), asumiendo una auténtica ética de la alteridad (Levinas, 2006); una ética que ha de cristalizarse en la búsqueda de unas relaciones humanas justas y en la toma de conciencia de los problemas existentes en nuestro entorno cercano y lejano, desarrollando la conciencia de que todos somos iguales de dignos y necesarios (Habermas, 1999). Es necesario, por tanto, el cultivo de identidades personales que aprendan a vincularse de manera fraternal con el resto de seres humanos, de forma que la solidaridad produzca, necesariamente, transformaciones personales y comunitarias.

Profundizando aún más en algunos de los valores que pueden ayudarnos a configurar la cultura de paz, consideramos muy importante el de la tolerancia. No una tolerancia en un sentido condescendiente o de simple respeto a las opiniones de los otros. Hablamos aquí de la tolerancia en el sentido en que lo hace Guichot (2012, p. 38): "Hay razones de orden superior para ser tolerantes, las morales: el respeto al otro, basado en su dignidad como ser libre, es decir, dotado de una autonomía que le hace capaz de determinar por sí mismo su forma de vida”. De esta forma entenderíamos la tolerancia como una virtud humana que rechaza cualquier forma de exclusión o explotación entre personas y busca nuevas y mejores formas de convivencia cada día (Cerezo, 2005). Estamos hablando en este caso de la concreción de una idea que no pretende quedarse en un concepto de tolerancia relacionado con la indulgencia o la condescendencia. Lo hacemos en relación a la capacidad de reconocer los derechos universales y las libertades fundamentales de los otros, promoviendo estilos educativos "que eduquen para la apertura y la disponibilidad y no para la cerrazón de lo seguro: de modo que la existencia se viva como un permanente desarrollo de nuevas y creativas posibilidades de verdad, belleza y bondad" (López, 2010, p. 88).

Por último, todas las características expuestas habrán de ser vertebradas por un modelo social de desarrollo, un modelo que dote a las personas de valor social, por encima de condiciones mercantiles o económicas. Un modelo inspirado en el "desarrollo a escala humana" (Elizalde, Max-Neff y Openhayn, 1986) que se oriente en buena medida a la satisfacción de las necesidades humanas, tratando de superar una visión economicista de la realidad y de la forma de relacionarnos para llegar o acercarnos a otra en la que el desarrollo económico dependa del desarrollo humano; asegurando condiciones de salud, educación y bienestar para todos de forma que influyan de manera positiva en un desarrollo armónico general de toda la sociedad.

Se trataría de promover un modelo social basado en una propuesta humanista (Krishnamurti, 2007) que facilite el aprendizaje de la vida en libertad, de personas libres que hacen uso de los bienes, pero no los convierten en un fin en sí mismos: "Un modelo de desarrollo en el que, aun existiendo relaciones de intercambio (mercado), se potencien las exigencias morales de redistribución (solidaridad) y reciprocidad por ser los tipos de relaciones humanas que realmente generan sociedad y comunidad" (Cáritas, 2009, p. 51).

En definitiva: al referirnos a la cultura de paz lo hacemos acerca de un proceso siempre inacabado de construcción y vivencia de valores como la solidaridad, la tolerancia y el respeto; promoviendo condiciones de justicia y posibilidades de participación social, de forma que todos los sujetos puedan ver satisfechas sus necesidades y se den posibilidades del aprendizaje de la vivencia de la vida en libertad, y en relación con los otros. 


\section{1. ¿Por qué una cultura de paz?}

La respuesta a esta pregunta es clara: porque debemos adquirir, si no lo hemos hecho ya, un compromiso con la dignidad de la persona y con los derechos humanos que den sustento a la misma. Pero para profundizar más en esta respuesta queremos recurrir al profesor Esteve (2010) y su idea de que educar es un compromiso con la memoria. Efectivamente, creemos que estos tres elementos ("educar", "compromiso" y "memoria") pueden ayudarnos a justificar la necesidad de una cultura de paz y una vivencia honda de los derechos humanos.

En lo que se refiere a la práctica educativa, no está de más comenzar recordando algo fundamental: la educación es unos de lo instrumento más valiosos para que la humanidad pueda encarar con garantías los múltiples desafíos a los que se enfrenta (Delors, 1994). Los rápidos y profundos cambios a los que asistimos, la gran cantidad de información que recibimos y no podemos procesar con tanta rapidez, la aldea global en que se ha convertido el mundo y que nos pone por delante realidades y problemas socioculturales de calado, nos sitúan ante la necesidad de saber entender, de aprender a dialogar, de buscar soluciones conjuntas.

En esta línea, creemos que la educación en derechos humanos es una herramienta a tener en cuenta en la promoción de la cultura de paz. No como una simple enseñanza de contenido cultural (Mestre, 2007), sino como una propuesta de vivencia de los valores que los derechos humanos nos ofrecen: "Suscitar situaciones educativas desde los derechos humanos, y aprovechar situaciones imprevistas para reconducirlas con una intencionalidad educadora centrada en estos derechos, exige pensar la educación desde el marco que nos proporcionan los derechos humanos" (Gil, 2008, p. 35). Son muchas las voces que nos señalan que entre la educación y los derechos humanos existe una relación bidireccional (Binaburo, 2012; Camps, 2007; Gil, 2008; Peces-Barba, 2007; etc.) por cuanto se da una imperiosa necesidad de enseñar y educar en derechos humanos, siendo la educación, a la vez, un derecho de la persona (López, 2005).

Actividades como la inclusión de los derechos humanos en todos los niveles de enseñanza; el análisis, la discusión y la reflexión de dichos derechos por parte de cada individuo junto con otros; utilizar metodologías activas y participativas en las que cada sujeto asuma su responsabilidad en la construcción del conocimiento a través de la interacción, la investigación, etc. podría dar lugar a intercambios pedagógicos eficaces en un marco de interculturalidad y de educación inclusiva: "los derechos humanos contribuyen decisivamente a resaltar parte de las bases éticas de la educación porque nos ayudan a comprender el significado humanizador de las finalidades educativas" (Gil, 2008, p. 36).

Otro de los componentes sobre los que queremos defender la importancia de la cultura de paz es el del compromiso, un compromiso con el ser humano y con un concepto de convivencia cívica (Camps, 2011) que haga unir esfuerzos tanto a la clase política como a la sociedad civil en la búsqueda y el mantenimiento de la paz: "que sea posible es tarea de todos" (Caride, 2013, p. 26). Es evidente la necesidad de comprometernos a todos los niveles con esta forma de entender al ser humano y la sociedad. Educación y política son conceptos íntimamente relacionados que se alimentan entre sí y han de caminar en una misma dirección, debiendo ser considerados como un asunto de estado (Jares, 2007) para mejorar las condiciones de vida de las personas y promover, así, una humanidad que sea radicalmente humana (Tamayo, 2012). Es necesario un compromiso político y un 
convencimiento pedagógico destinados a una construcción de esa cultura, de esta forma de entender las relaciones humanas y las sociedades. En estos tiempos de crisis, recuperar y apostar firmemente por valores que nos hagan más humanos, libres, solidarios, etc. es un reto que compete a todos: desde aquellos encargados de promulgar leyes y hacerlas cumplir hasta cualquier ciudadano de a pie. Este compromiso es una responsabilidad común.

Por último, queremos hacer referencia a la memoria como un factor que ha de ayudarnos a explicar por qué es necesaria una cultura de paz: "Educar es un compromiso con la memoria; con nuestra memoria individual y con la memoria colectiva” (Esteve, 2010, p.18). Recordar y reflexionar sobre el pasado para comprobar que los derechos humanos son conquistas sociales conseguidas con el esfuerzo y la vida de personas determinadas; que, a lo largo y ancho del tiempo y de la historia, se han dado acontecimientos que nos indican las grandezas de la especie humana y sus miserias; y que de unas y otras podemos y debemos aprender para repetirlas o desterrarlas de nuestras acciones, son aspectos importantes a tener en cuenta: "A veces, vale la pena volver la vista hacia el pasado para saber si nuestro mundo actual avanza o retrocede, y cuáles de los valores de moda deben ser combatidos desde la educación “ (p. 17).

\subsection{La cultura de paz en los centros educativos. Proyecto "Red Escuela: Espacio de Paz"}

En el año 1995 se dio un impulso en Andalucía a la "Red Escuela: Espacio de Paz" (Orden 19 de diciembre de 1995), por medio de una Orden en diciembre de ese mismo año. Dentro de dicha orden la educación moral y cívica adquiría una importante significatividad, tratando de buscar un cambio de mentalidad social que colaborara en el abordaje educativo de los problemas de convivencia, que se nos presentan como un gran reto tanto a nivel social como en los propios centros escolares.

Dicho cambio de mentalidad se empezó a concretar en el curso 2002-2003, en un proyecto pionero en el que comenzaron trescientos centros andaluces y en el que en la actualidad están incluidos casi dos mil. Para acogerse a este proyecto se debe desarrollar un programa con vigencia de dos cursos escolares que trate los siguientes contenidos:

- El aprendizaje de una ciudadanía democrática.

- La educación para la paz, los derechos humanos y la democracia.

- La mejora de la convivencia escolar y la resolución pacífica de los conflictos.

- La prevención de la violencia.

Se trata de un proyecto destinado a toda la comunidad educativa en su máxima expresión: profesorado, alumnado, familias, personal no docente, etc., apostando por un centro educativo como espacio de encuentro de personas y entidades de muy distinta naturaleza, donde el compromiso, el esfuerzo y el intercambio de ideas debe contribuir a un clima de relaciones favorables para todas las partes y a todos los niveles, aportando al individuo conceptos positivos para su desarrollo integral.

Existen dos modalidades para el proyecto: Unicentro, desarrollado en un único centro; e inter-centro, llevado a cabo por varios centros, destacándose la primera opción como la principal, representando casi el $82 \%$ de los centros incluidos en el proyecto según datos de la Resolución Provisional de 1 de septiembre de 2011 por la que se reconocen centros 
docentes pertenecientes a la Red Andaluza "Escuela: espacio de Paz" como Centros Promotores de Convivencia Positiva. Binaburo (2012) sostiene que los principales aciertos del proyecto han sido:

- Desarrolla una metodología de investigación -acción.

- Se transforma el rol del educador a través de iniciativas compartidas, creativas y realistas.

- Abre la participación de agentes externos a la comunidad educativa.

- Se modifica la visión de conflicto.

- Supone un reto cívico.

- Se ha fomentado y facilitado la formación del alumnado, profesorado y familias en el ámbito de la cultura de paz, la prevención y la resolución de conflictos.

- El trabajo en red ha permitido la difusión de las buenas prácticas de convivencia y cultura de paz, así como de materiales, desarrollados por los centros, para que estén a disposición de todos los centros de la red.

Para participar en la red existen una serie de requisitos (Orden de 11 de abril de 2011): a) estar aprobado por el Consejo Escolar, a propuesta del Profesorado. b) Contar con un docente responsable de la coordinación de las actuaciones derivadas de la participación en dicha red. c) Contar con un equipo de profesorado implicado en las propuestas de mejora y en la realización de las actividades que se planifiquen.

Nos centraremos en nuestra investigación en un centro de Educación Secundaria de Málaga, un centro con 376 alumnos, con un ratio de 25 y 32 alumnos en las doce unidades que tiene establecidas. En torno al $90 \%$ de este alumnado continua estudios postobligatorios, aunque un significativo número se dirige a la formación profesional.

El centro participa en el proyecto "Escuela: Espacio de Paz" desde el curso 2003-2004 y en estos momentos el proyecto que desarrolla recibe el nombre de "Convivir en la diversidad”, trabajando de forma conjunta la Cultura de paz y la Coeducación.

Nuestros objetivos de trabajo al investigar han sido:

- Conocer en qué medida se está viendo implementada la cultura de paz en el centro, por medio del aula de convivencia.

- Conocer la concepción del alumnado que acude al aula de convivencia del centro acerca de dicha aula.

- Analizar cuál es el concepto que del término "conflicto" tiene el alumnado del centro.

- Ofrecer algunas líneas concretas de trabajo que ayuden a optimizar la implantación de la cultura de paz en el centro.

\section{Método}

El estudio que presentamos se basa en un diseño mixto (Brannen, 2005), tratando de recolectar, analizar y vincular datos cuantitativos y cualitativos en el mismo estudio o en 
una serie de investigaciones, mezclando la lógica inductiva y deductiva (Teddlie y Tashakkhori, 2003). La realidad tan diversa en la que queríamos realizar nuestra investigación nos animaba a utilizar este tipo de metodología. Dentro de la misma decidimos utilizar también la observación no participante (Rodríguez y Gil, 1996) en la que la detección de necesidades fue el objetivo principal. Esta etapa la desarrollamos durante dos semanas, en las cuales nuestra actividad consistió en tratar de conocer todos los aspectos del centro relacionados con la organización y la gestión de la Cultura de Paz.

En definitiva, optamos por un diseño de estas características ya que entendíamos que podría ayudarnos a lograr una perspectiva más precisa de la realidad, siendo un forma óptima de estudiar relaciones dinámicas y sumamente intrincadas (León y Montero, 2003), aspecto clave de todo estudio relacionado con el ser humano.

\subsection{Muestra y diseño}

La elección de nuestra muestra tiene una clara intención que no es otra que la de dar la voz (Rudduck y Flutter, 2007) a los auténticos protagonistas del trabajo educativo: el alumnado. Es necesario atrevernos a mirar a los estudiantes con otros ojos, admitiendo que hay aspectos que competen a su realidad que hasta ahora no les hemos consultado con suficiente seriedad y que es necesario comenzar a hacerlo (Sheeh et al., 2005) para lograr que todas nuestras áreas de trabajo adquieran mayores dosis de calidad educativa. Sin la voz del alumnado, las investigaciones pierden sustrato pedagógico y corren el riesgo de mostrar una información que olvida a los destinatarios de la tarea educativa (Sandoval, 2011).

Nuestra investigación es de carácter exploratorio y descriptivo (Hernández, Fernández y Baptista, 2006). La recogida de datos tuvo lugar en el curso académico 2012-2013 con una muestra intencional de 206 sujetos repartidos en 117 ( 57 chicos y 60 chicas) de $1^{\circ}$ de ESO y 89 (56 chicos y 33 chicas) de $2^{\circ}$ de ESO, dentro del centro al que hemos hecho referencia anteriormente. La elección de estos sujetos se debe a que son los grupos donde se concentran un mayor número de hojas de incidencias en el centro, según se desprende del análisis que pudimos hacer de las mismas. Y es que tal y como se ha señalado, decidimos también analizar las hojas de incidencia que cumplimentaban los alumnos, tutorizados por los docentes responsables del Aula de Convivencia, ya que entendíamos que nos aportaban una información que podría ser de gran valor.

\subsection{Instrumento de recogida de información}

Los instrumentos que utilizamos para recabar la información fueron dos: por un lado elaboramos un cuestionario ad hoc de 20 preguntas agrupadas en tres bloques fundamentales:

- Las ocho primeras destinadas a conocer si el alumnado sabe de la existencia del proyecto Escuela Espacio de Paz en el centro: asignaturas, responsables del proyecto, actividades, etc. por ejemplo: “ ¿Conoces el proyecto Escuela Espacio de paz?" “Consideras importante que se desarrolle un proyecto así en tu centro?"

- Las cuatro siguientes destinadas a conocer la valoración que el alumnado hace sobre el proyecto, utilizando una escala tipo Likert, por ejemplo: "Asigna un valor del 1-10 (10 máximo) de importancia del proyecto". 
- Las restantes preguntas, planteadas de forma abierta, estaban destinadas a conocer la valoración que el alumnado hacía del Aula de Convivencia y de conceptos como "paz” o "conflictos”: “PPara qué sirve el Aula de Convivencia?”, "Define con una palabra el término "conflicto" o "Realiza propuestas para el Proyecto".

Para la elaboración de este cuestionario decidimos seguir los pasos que autores significativos en la materia (Castillo y Cabrerizo, 2003; Fernández, 2001; Pérez, 2004; etc.) nos ofrecen para construir herramientas fiables: planificar la construcción del cuestionario definiendo objetivos, definir claramente la información que se desea obtener, elegir la muestra, elaborar el contenido de acuerdo a la información que queremos, etc. Además, todo el procedimiento investigador fue evaluado positivamente por un tribunal de TFM del Máster de Cultura de Paz, que valoró de manera adecuada el trabajo metodológico.

Por otro lado, también contamos como instrumento de recogida de información con la hoja de incidencias que debían rellenar el alumnado enviado al Aula de Convivencia y ser firmada por el docente responsable. Dicha hoja fue elaborada por el Equipo de Orientación del IES y consta de cinco preguntas:

- Describe qué ha ocurrido

- ¿Por qué has actuado de esta manera?

- ¿Cómo me siento?

- ¿Qué puedo hacer para resolver esta situación?

- ¿A qué me comprometo?

El análisis de ambos documentos tenía presente tanto aspectos cuantitativos como cualitativos, desde la cuantificación del número de hojas de incidencia dentro de muy diversas categorías, como curso, grupo o sexo; hasta valoraciones cualitativas relacionadas con el Aula de Convivencia: causas por las que habían sido enviados a la misma, opiniones expresadas en relación al proyecto por parte de los jóvenes, etc.

\subsection{Categorías de análisis}

Para el análisis de las hojas de incidencia, dada la multiplicidad de respuestas, resultaba imprescindible la formalización de un sistema de categorías que nos sistematizara, en la medida de lo posible, el gran volumen de información con el que trabajamos. Este sistema fue creado únicamente para el primer ítem de la hoja de incidencia, en el que se pedía al alumno o alumna que explicara qué había ocurrido, aspecto que dotaba al mismo un carácter abierto. En cambio, no fue necesario para los restantes ítems ya que se trataban principalmente de preguntas cerradas.

Se localizaron veintiuna respuestas distintas para la explicación de las razones por las que el alumno o alumna en cuestión había sido mandado al Aula de Convivencia. Así, se establecieron las tres siguientes categorías principales, además de una adicional denominada "No justifica". Exponemos algunos ejemplos de subcategorías para facilitar al lector la comprensión de las mismas:

- Interrupciones: "Levantarse sin permiso", "No traer el material", "Comer chicle”... 
- Faltas de respeto: "Molestar a los compañeros", "Desobedecer", "Usar un tono o expresión inadecuado"...

- Violencia: “Insultar”, "Peleas”, "Pegar”...

Por otro lado, en los cuestionarios no fue necesaria la elaboración de un sistema de categorías específico, únicamente por curso y por sexo. Las razones de esta decisión fueron básicamente dos: en primer lugar, por motivos de espacio hemos decidido no incluir todas las respuestas aportadas por los alumnos, destacando aquellas que entendemos son más significativas y puedan aportar información sustanciosa a nuestro análisis. En segundo lugar, las respuestas que obtuvimos fueron fácilmente clasificables en categorías como "Discusiones", "Castigar", "Problemas", etc.

\section{Resultados}

En cuanto a los resultados, presentamos, en primer lugar, la información referente al cuestionario, concretamente para los ítems en los que se les pregunta qué es el Aula de Convivencia, que realicen una definición del término "conflicto" y, por último, que hagan propuestas de actividades para mejorar el proyecto.

Por otro lado, también presentaremos el análisis realizado de las hojas de incidencia, sólo para su primer ítem que, como indicamos anteriormente, pide al alumno o alumna los motivos por los que han sido enviados al Aula de Convivencia.

\section{1. ¿Qué es el aula de convivencia?}

Las respuestas aportadas en este ítem nos muestran que casi tres de cada cuatro sujetos definen este aula como un "aula de castigo", siendo un resultado muy contundente y que cuestiona desde su raíz la implementación real del proyecto en el centro, ya que sólo un $17.42 \%$ responden que se trata de un aula de reflexión (figura 1).

Además, destacan algunas respuestas del estilo "Es un aula donde van los niños que molestan" o "Es un aula donde van niños que no trabajan", lo que nos lleva a situarnos en la idea de que nos encontramos en un espacio que busca únicamente reducir conductas, minimizando la posibilidad de que su uso sea destinado a la construcción de convivencia y el aprendizaje de vivir en común.

Uno de los aspectos más llamativos lo encontramos en el ítem siguiente, donde se les preguntaba por la función del Aula de Convivencia, a lo que contestan, en un 42.78\%: "Para reflexionar". Es decir, que se aprecia un número significativo de respuestas en la que se sostiene que "El Aula de Convivencia es una aula de castigo que sirve para reflexionar", lo que nos señala que parece existir una idea teórica positiva acerca del sentido del trabajo del proyecto de Cultura de Paz, pero que no se ve reflejada en la realidad, que nos ofrece una praxis completamente alejada de propuestas pedagógicas. Creemos que las respuestas nos indican una confusión importante al expresar que un espacio que tiene la intención de ser de aprendizaje de la convivencia esté relacionado con la idea de un "aula de castigo" para la reflexión. Desde nuestro punto de vista, los términos "castigo" y "reflexión" tienen muy poca relación si hablamos desde una óptica educativa, de una praxis transformadora. 


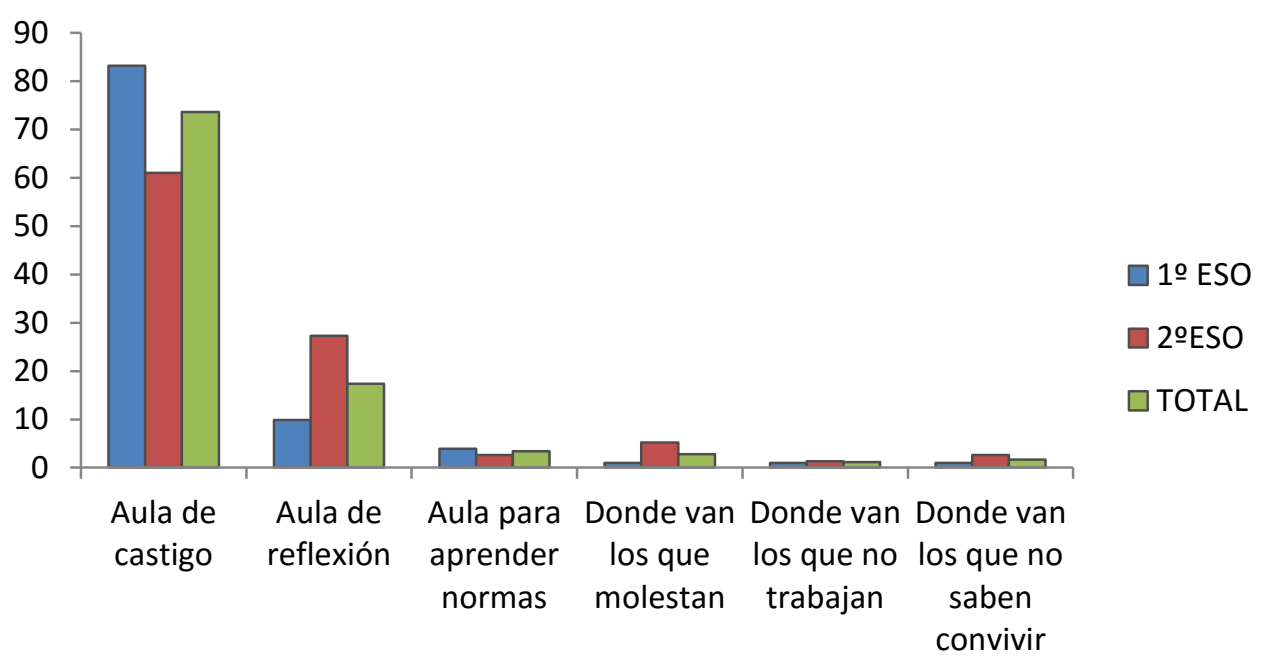

Figura 1. Respuestas de los alumnos a la pregunta qué es el Aula de convivencia Fuente: Elaboración propia.

Esta tendencia viene corroborada por el resto de respuestas que ofrece el alumnado en relación al funcionamiento del Aula de convivencia como "Para corregir comportamientos" (11,11\%), "Para castigar" (26,67\%), o incluso "Para nada" con un $2.22 \%$.

El análisis de estos datos nos señalan que el Aula de convivencia parece cumplir de forma deficiente con el rol y la responsabilidad que debería asumir, un rol en el que el centro escolar “(...) sea capaz de promover en su alumnado las ganas de aprender. Todo aquello que la escuela actual explique dentro de sus aulas debe ser reconocido por el alumnado como algo próximo a él" (Pallarés, 2014, p. 62). Si los alumnos enviados al Aula de convivencia la entienden, en su gran mayoría, como un espacio de castigo, ajeno a su realidad personal, podemos afirmar consecuentemente que existen deficiencias significativas en esta tarea.

\subsection{Define en una palabra el término "conflicto"}

Vamos a conocer en este momento cómo definen los alumnos implicados en el Aula de Convivencia el término conflicto. Solemos tener una visión negativa de dicho término, asociándolo a la violencia y a situaciones no resueltas. Tal y como hemos venido defendiendo desde el principio, para la Cultura de Paz dicha situación puede ser una oportunidad para producir transformaciones sociales, construir juntos otro tipo de relaciones, aprender a analizar situaciones sin uso de la violencia, etc. Los datos que hemos podido recabar al preguntar a los sujetos acerca del término conflicto aparecen en la figura 2.

Las respuestas recogidas vienen a reforzar, de algún modo, la tendencia antes expuesta. Únicamente un $10 \%$ del total entienden el conflicto como un "intercambio de ideas" o un "desacuerdo", expresiones que podríamos considerar como positivas en relación al conflicto. El resto de respuestas, más del $80 \%$, ofrecen un carácter evidentemente peyorativo: "Pelea", "Guerra", "Violencia", "Problema", "Enfrentamiento" o "Amargura". Entendemos que estos datos nos indican que el concepto de conflicto que los sujetos preguntados entienden está aún lejos de lo que desde la Cultura de Paz se defiende: uno 
en el que se aproveche el conflicto como medio para el aprendizaje de la vivencia en común y de valores democráticos.

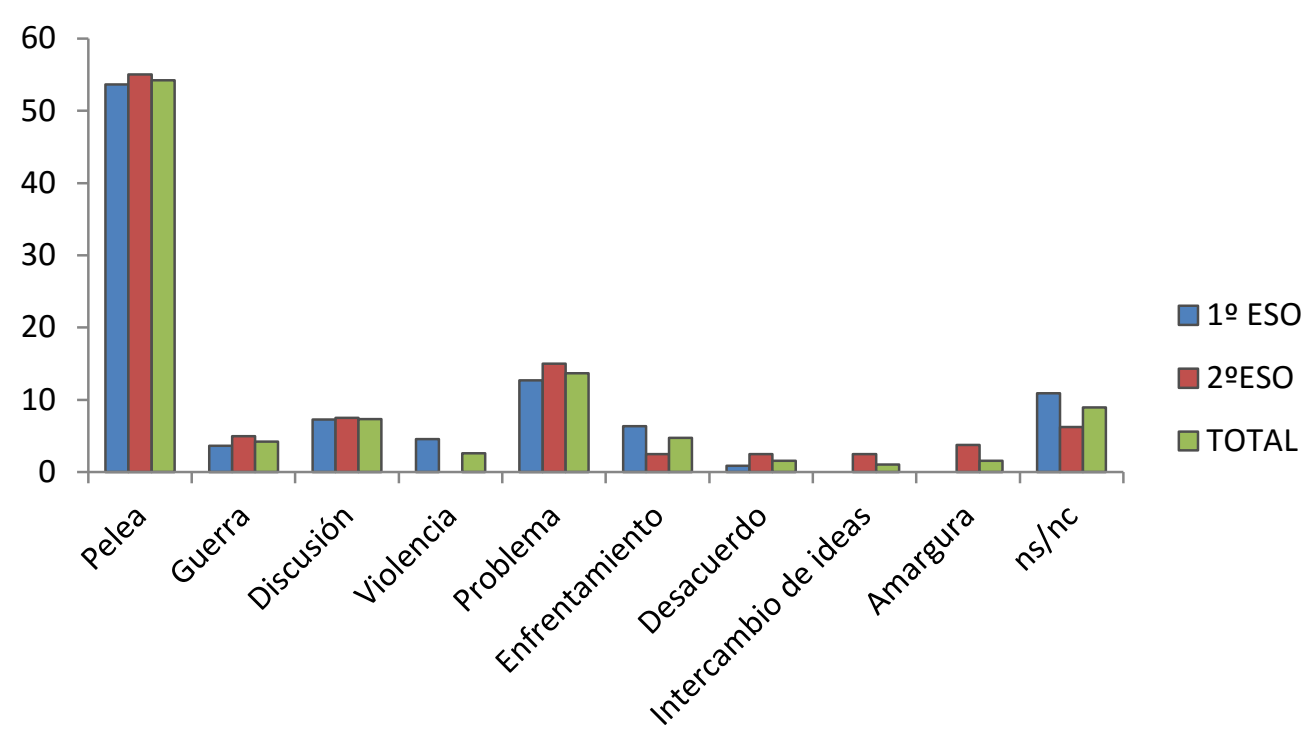

Figura 2. Respuestas de los alumnos a la definición del término "Conflicto" Fuente: Elaboración propia.

Gestionar de forma positiva situaciones de conflicto en un Centro Escolar no es tarea fácil, necesita la implicación y la responsabilidad de toda la comunidad educativa y una vivencia común de valores. El espíritu de un proyecto como el de "Proyecto Escuela: Espacio de Paz" es el de construir conjuntamente una cultura democrática, en el más profundo sentido de esta expresión. El hecho de que los chicas y chicas encuestados definan como lo hacen el término "conflicto" nos está indicando que la vivencia en la cultura de paz en el Centro está aún en una fase inicial, un momento en el que cuestiones tan importantes para dicha cultura se encuentran poco arraigadas en el imaginario común.

Pasamos ahora, para terminar el análisis del cuestionario, a conocer las actividades que los propios alumnos y alumnas proponen para el proyecto.

\section{3. ¿Qué actividades se podrían realizar para el desarrollo del proyecto?}

Una de los objetivos de nuestra investigación ha sido en todo momento el de tratar de escuchar y dar protagonismo a los destinatarios de todo el trabajo educativo: en este caso los propios alumnos y alumnas del Centro.

Para ello, y dentro de una pregunta de nuestro cuestionario, decidimos pedirles que hicieran propuestas que, desde su punto de vista, pudieran mejorar el desarrollo del proyecto (figura 3 ).

Dentro de las respuestas del alumnado se destacan dos grupos principalmente: uno que responde "no sabe/ no contesta"; y otro, mayoritario que responden: "actividades grupales". Incluso en esa línea se destacan respuestas del estilo de "talleres", "actividades solidarias" o incluso "videos".

Asumiendo la existencia de una parte significativa de respuestas que eligen la opción de "no sabe/no contesta", creemos que es algo muy destacable y a tener en cuenta, las 
respuestas que los alumnos ofrecen; respuestas que tienen que ver con actividades de encuentro entre iguales y de relación, espacios donde poder compartir tiempo junto a otros de forma útil y constructiva. Propuestas, al fin y al cabo, en la línea de lo que se proponen como medios para facilitar la Cultura de Paz: "Una comunidad democrática debe instituir prácticas de valor que faciliten la cooperación entre sus miembros y la experiencia de valores encarnados en formas de comportamiento" (Puig, 2000, p. 187).

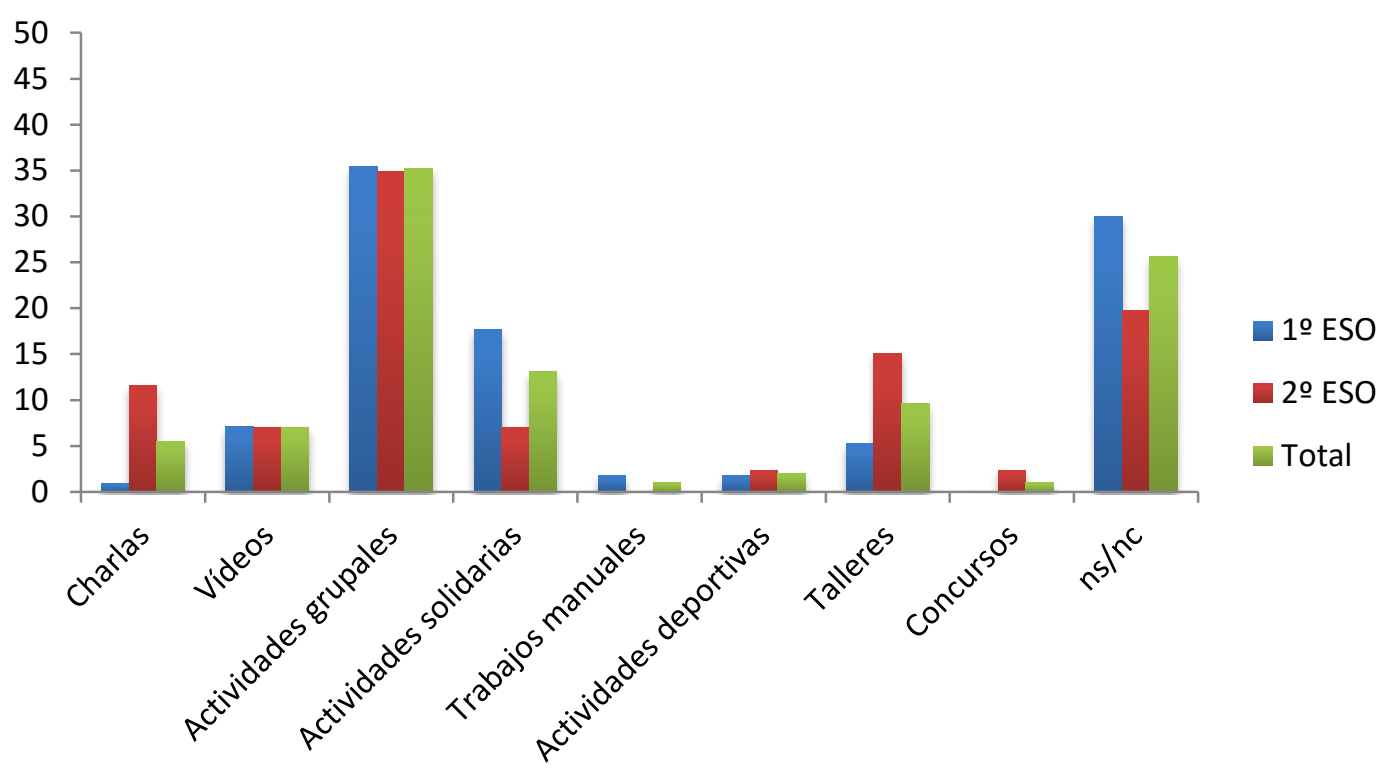

Figura 3. Propuestas de actividades de los alumnos Fuente: Elaboración propia.

\subsection{Análisis de las hojas de incidencia}

Cabría destacar que la categorización de la hoja de incidencias fue un tanto complicada, ya que la mayoría de las subcategorías podían entenderse como "Interrupciones"; sin embargo, tratando de equiparar las principales categorías y haciendo un análisis profundo de su significado, categorías como "No respetar el material", "Sentarse mal", "Mirar por la ventana", se incluyeron en "Faltas de respeto".

El estudio de los datos nos indica que en un $65.85 \%$ de las ocasiones, los estudiantes son enviados al Aula de Convivencia por motivos referidos a "Interrupciones" (Figura 4). Acuden a dicha Aula aquellos sujetos que tienen una conducta contraria a la convivencia en el grupo y/o en el centro. Se invita a la reflexión de si conductas como "No traer el material", "Levantarse sin permiso" o "Comer chicle" son contrarias a la convivencia de un grupo de personas. Llaman la atención estos datos: más de la mitad de los alumnos son enviados al Aula de Convivencia por mantener una serie de conductas que, más que contrariar la convivencia, parecen contrariar a una parte de la comunidad educativa que utiliza dicho espacio para mantener una situación de privilegio y poder.

Respecto a las conductas pertenecientes a las categorías "Faltas de respeto", lo llamativo del caso es que tanto las causas referentes a esta categoría como a "Violencia" oscilan en torno al 15\%; apreciándose una diferencia muy reseñable frente al porcentaje que presenta "Interrupciones", un 65,85\%. 
Por otro lado, y aunque sea un porcentaje muy pequeño, sólo un 3,14\%, en la categoría "No justifica" también podemos extraer una interesante información, ya que deberíamos reflexionar sobre qué sentido tiene un Aula de Convivencia y un proyecto de las dimensiones como "Escuela: Espacio de Paz" si se dispone de una hoja de autorreflexión, que debe ser leída y firmada por un docente cuando el alumno o alumna haya reflexionado, al menos, sobre qué ha ocurrido; rellenándose finalmente, en un buen número de casos, como si de un formulario se tratase.

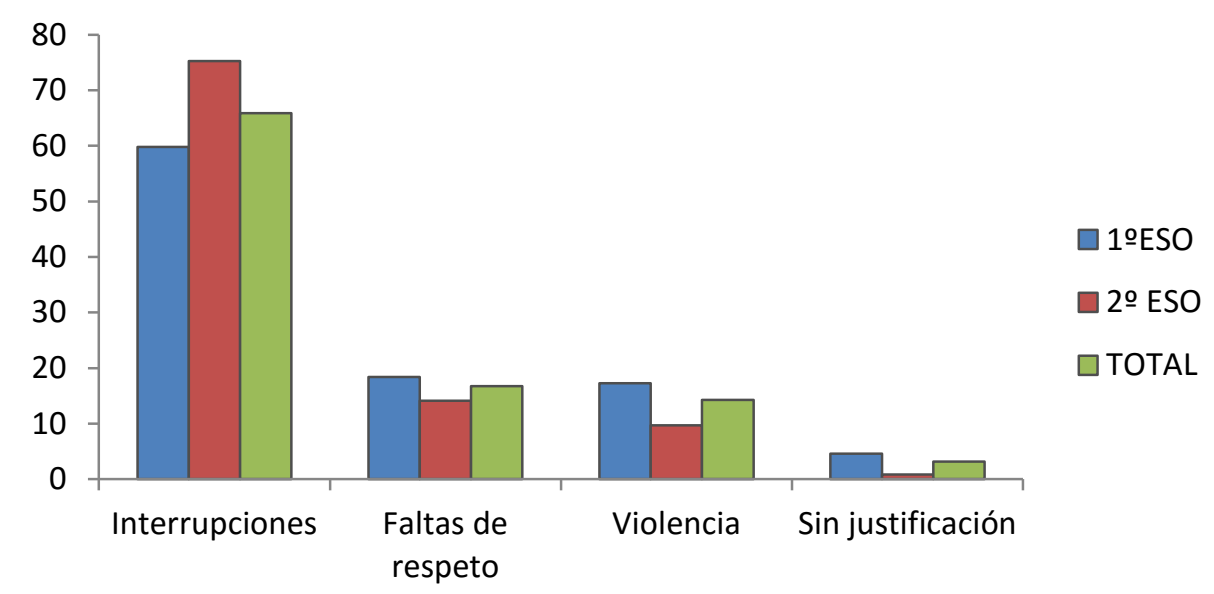

Figura 4: Motivos por los que los alumnos han sido enviados al Aula de convivencia Fuente: Elaboración propia.

\section{Discusión y Conclusiones}

Los datos presentados y analizados son, en buena medida, concluyentes. Entendemos que nuestro estudio se circunscribe a una realidad muy determinada, y no pretendemos extrapolar nuestros datos y conclusiones hacia el resto de realidades como algo inapelable, sino ofrecer algunas reflexiones y evidencias que nos ayuden a todos a continuar creciendo de forma significativa en la implementación de la cultura de paz.

Dicho esto, y en referencia al Centro analizado, los alumnos y alumnas estudiados poseen un concepto del Aula de Convivencia muy relacionado con un espacio punitivo, destacándose conceptos e ideas como "corregir", "castigar", etc. A pesar de eso, también podemos afirmar que entienden el sentido teórico del proyecto ("para reflexionar", señalan), pero están llamando nuestra atención acerca de una situación preocupante: el medio utilizado no está cumpliendo con las funciones que se le deberían suponer, ofreciendo un espacio que se vive de forma negativa, lejos de propuestas educativas. El alumnado parece sufrir las consecuencias de un proceso que no funciona como se le supondría. Los datos expuestos nos indican una situación deficitaria respecto a lo que deberíamos esperar de un proyecto de este calado. Se intenta ofrecer a los alumnos aprendizajes de diversa índole y tipo, pero existen graves vacíos en cuanto al aprendizaje de la convivencia y la paz, optándose por un aprendizaje basado más en el condicionamiento que en lo verdaderamente educativo (Esteve, 2003); poniendo en cuestión, desde nuestro punto de vista, la necesidad educativa de la existencia del Aula de Convivencia, que únicamente está siendo utilizada como medio represor sin ofrecer más posibilidades. 
Todo lo dicho viene a confirmarse al preguntar a los sujetos acerca de su concepto de la palabra "conflicto". En este caso la connotación negativa es mayor que respecto al Aula de Convivencia: "pelea", "guerra", "amargura" son las respuestas más destacadas que se nos dan. Desde nuestro punto de vista, estas concepciones nos indican la necesidad de un cambio en el enfoque del trabajo, un cambio hacia una auténtica formación del alumnado y con él de toda la comunidad educativa. Resulta de vital importancia dotar al alumnado de una serie de herramientas que los coloquen en las mejores condiciones para una interacción óptima con su entorno; para un desarrollo de habilidades relacionales y capacidades que los lleve a un aprendizaje permanente a lo largo de su vida (Mezirow, 2000).

El análisis de la realidad que nos ocupa nos indica que el desarrollo de la Cultura de Paz en el Centro se encuentra en una fase inicial: los principios de dicho proyecto parecen ser compartidos por la amplia mayoría de los implicados en nuestra investigación, aunque la implementación en la vida diaria de los alumnos y alumnas esto no sea así. Como hemos señalado, se utiliza el Aula de Convivencia como forma de control hacia aquellos alumnos que no encajan en la dinámica de las aulas, negándoles la posibilidad de participar y hacer aportaciones.

Entendemos necesario devolver el protagonismo al alumnado para que colabore en la creación del currículum, generando conocimiento. En este sentido, el aprendizaje dialógico (Freire, 1996), actividades dirigidas al desarrollo de la empatía hacia los demás, la aceptación de lo diferente, y el hecho de promover formas participativas de aprendizaje como la escucha, la lectura compartida de textos, creación de materiales, cine-fórum, experimentación, etc. serán actividades que ayudarán a profundizar en esta línea educativa (García, 2013).

Creemos que es un hecho claro que los niños no aprenden de nosotros, sino que nos aprenden a nosotros (Martínez, Rodríguez y Romera, 2004). Siguiendo esta línea, resulta una obviedad que si se pretende alcanzar los objetivos de este proyecto acometamos todos los agentes educativos un compromiso mayor y mejor a favor de la Cultura de Paz. Para ello, entendemos necesario un trabajo conjunto de toda la comunidad educativa (Traver, Sales y Moliner, 2010), en el que se produzca una dinámica continua, no fragmentada, generando cauces de participación, compromiso y responsabilidad de todos estos agentes. Así, proponemos desarrollar actividades asamblearias que faciliten la implementación de un modo más real del proyecto "Escuela: espacio de paz" a partir de una visión más comunitaria, que responsabilice a todos.

Sostenemos que la educación para la paz debe investigar acerca de la forma de acompañar a cada persona en la realización de análisis de su realidad y del mundo en el que vive, promoviendo reflexiones, pensamientos y actividades que han de poder concretarse en la vida diaria, generando un conocimiento aplicable y útil que nos facilite la posibilidad de encontrar, cada día, nuevas y mejores formas de convivencia, desarrollo personal y construcción comunitaria.

\section{Referencias}

Aranguren, L. (2002). Educar en el compromiso. Valores para vivir en sociedad. Madrid: PPC.

Binaburo, J.A. (2012). Educar desde el conflicto: guía para la mediación escolar. Madrid: CEAC. 
Brannen, J. (2005). Mixing methods: qualitative and quantitative research. Londres: Routledge.

Castillo, S. y Cabrerizo, J. (2003). Evaluación de programas de intervención socioeducativa: agentes y ámbitos. Madrid: Pearson Educación.

Camps, V. (Coord.) (2011). Civismo. Barcelona: Editorial Proteus.

Caride, J.A (2013). Educar en la convivencia: la cultura de paz como construcción pedagógica y social. En M.T. Castilla, V.M. Martin, A.M. Sánchez y E.S. Vila. (Coords.), Cultura de paz para la educación (pp.17-31). Madrid: GEU.

Cáritas (2009). Modelo de acción social. Madrid: Cáritas Española Editores.

Cerezo, P. (2005). Democracia y virtudes cívicas. Madrid: Biblioteca nueva.

Delors, J. (1994). La educación encierra un tesoro. Madrid: Santillana-UNESCO.

Esteve, J.M. (2003). La tercera revolución educativa: la educación en la sociedad del conocimiento. Barcelona: Paidós.

Esteve, J.M. (2010). Educar: un compromiso con la memoria. Barcelona: Octaedro.

Elizalde, A., Max-neff, M. y Openhain, M. (1986). Desarrollo a escala humana, una opción para el futuro. Santiago: Cepaur.

Flecha, R. (2006). ¿Qué cambiará en las escuelas cuando volvamos a Freire? En I. Alcalde (Ed.), Transformando la escuela: comunidades de aprendizaje (pp. 13-18). Barcelona: Graó.

Freire, P. (1996). Pedagogía de la autonomía. Saberes necesarios para la práctica educativa. México: Siglo XXI.

García, E. (2013). Hacia una forma más holística y dialógica de educar para la paz. En M.T. Castilla, V.M. Martin, A.M. Sánchez y E.S. Vila (Coords.), Cultura de paz para la educación (pp. 93-109). Madrid: GEU.

Gil, F. (2008). Ciudadanía y humanidad. La educación en el disenso. Teoría de la Educación, Revista Interuniversitaria, 20, 25-44.

Gimeno, J. (2001). Educar y convivir en la cultura global. Madrid: Morata.

Guichot, V. (2012).Tolerancia, una virtud cívica clave en una educación para la ciudadanía activa, compleja e intercultural. Bordón. Revista de Pedagogía, 64, 35-47.

Habermas, J. (1999). La inclusión del otro. Barcelona: Península.

Hernández, R., Fernández, C. y Baptista, P. (2006). Metodología de la investigación. México: McGraw-Hill.

Jares, X.R. (2007). Pedagogía da convivencia. Oporto: Profediçöes.

Krishnamurti, J. (2007). La educación y el significado de la vida. Madrid: Edaf.

Leon, O. y Montero, L. (2003). Métodos de investigación en psicología y educación. Madrid: McGrawHill.

Levinas, E. (2006). Humanismo del otro hombre. Madrid: Siglo XXI.

López, J.A. (2010). ¿Qué “yo” es valioso para el mundo de hoy? Teoría de la Educación, Revista Interuniversitaria, 22, 65-90.

López, P. (2005). Educación en derechos humanos: suspenso. En L.M. Naya. (Coord.), La educación y los derechos humanos (pp.155-173). Donostia: Erein.

Martínez, O., Rodríguez, J. y Romera, M. (2004). Técnicas de dinámicas de grupo: un recurso para la organización del clima social en el aula. Granada: Ediciones Adhara, S.L. 
Mayor, F. (2011). Donde no habite el miedo. Madrid: S.A. Litoral.

Max-Neff, M. (1998). Desarrollo a escala humana. Barcelona: Nordan-Comunidad.

Mestre, J.V. (2007). La necesidad de la educación en derechos humanos. Barcelona: UOC.

Mezirow, J. (2000). Learning as transformation. Critical perspectives on a theory in progress. San Francisco, CA: Jossey-Bass.

Muñoz, F.A. (2001). La paz imperfecta. Granada: Universidad de Granada.

Muñoz, F.A. y López, M. (1999). La paz en la historia. Granada: Eirene.

Pallarés, M. (2014). El legado de Paulo Freire en las escuelas de hoy. De la alfabetización crítica a la alfabetización en medios de comunicación. Teoría de la Educación, Revista Interuniversitaria, 26, 59-76. doi: 10.14201/teoredu20142615976

Pérez, G. (2004). Modelos de investigación cualitativa en educación social y animación sociocultural. Aplicaciones prácticas. Madrid: Narcea.

Rodríguez, G. y Gil, J. (1996). Metodología de la investigación cualitativa. Bilbao: Aljibe.

Rudduck, J. y Flutter, J. (2007). Cómo mejorar tu centro escolar dando la voz a tu alumnado. Madrid: Morata.

Ruiz, C. (2008). Educación y derechos humanos desde la participación y la convivencia. Madrid: Fundación SM.

Sandoval, M. (2011). Aprendiendo de las voces de los alumnos y alumnas para construir una escuela inclusiva. REICE. Revista Iberoamericana sobre Calidad, Eficacia y Cambio en Educación, 9(4), 114-125.

Sánchez, S. (2012). La perspectiva de la cultura de paz en el estudio de la diversidad cultural y la convivencia en educación. En M.T. Castilla y V.M. Martín (Coords.), Buenas prácticas en educación intercultural y mejora de la convivencia (pp.25-35). Madrid: Wolters Kluwer.

Sheehy, K, Nind, M. y Simmons, K. (2005). Ethics and research in inclusive education. Londres: Routledge Falmer.

Tamayo, J. (2012). Invitación a la utopía: estudio histórico para tiempos de crisis. Madrid: Trotta.

Traver, J.A., Sales, A. y Moliner, O. (2010). Ampliando el territorio. Algunas claves sobre la participación de la comunidad educativa. REICE. Revista Iberoamericana sobre Calidad, Eficacia y Cambio en Educación, 8(3), 96-119.

Teddlie, C. y Tashakkori, A. (2003). Handbook of mixed methods in social E behavioral research. California: Sage.

Tuvilla, J. (2004). Cultura de paz. Fundamentos y claves educativas. Bilbao: Desclée de Brouwer. 